\title{
Effect of Unidirectional Shear on the Structure of Triblock Copolymers. 2. Polystyrene-Polyisoprene-Polystyrene
}

\author{
Faith A. Morrison ${ }^{1}$ and H. Henning Winter* \\ Department of Chemical Engineering, University of Massachusetts, \\ Amherst, Massachusetts 01003

\section{Wolfram Gronski} \\ Institut für Makromolekulare Chemie, Universität Freiburg, 7800 Freiburg, \\ Federal Republic of Germany \\ John D. Barnes \\ National Institute of Standards and Technology, Gaithersburg, Maryland 20899 \\ Received February 20, 1990
}

\begin{abstract}
Rheological and small-angle X-ray scattering (SAXS) measurements on a polystyrenepolyisoprene-polystyrene block copolymer indicate that high-strain, high-stress unidirectional shear flow in the microphase-separated regime produces a well-oriented, microdomain structure with cylindrical microdomains. Experiments in low-stress, high-strain flow produced little orientation. The material studied is a high molecular weight copolymer with $24 \mathrm{vol} \%$ polystyrene and a cylindrical microdomain morphology as indicated by SAXS. Flow above the microphase-separation temperature $\left(T_{\mathrm{MST}}\right)$ determined by linearviscoelastic measurements resulted in no microdomain orientation. The linear-viscoelastic results show that materials sheared near $T_{\text {MST }}$ do not recover to a well-microphase-separated morphology after high-strain shear flow. A flow mechanism for microphase-separated block copolymers proposed previously is compatible with the results obtained here.
\end{abstract}

\section{Introduction}

In the first paper of this series ${ }^{2}$ the effect of unidirectional shear flow on a polystyrene-polybutadienepolystyrene block copolymer, SBS81, was investigated. That polymer showed evidence of microphase separation at all temperatures studied, indicating that the microphaseseparation temperature, $T_{\mathrm{MST}}$, is much greater than 220 ${ }^{\circ} \mathrm{C}$. This study concerns a second polymer of comparable molecular weight and polystyrene (PS) volume fraction, which contains a different centerblock, polystyrenepolyisoprene-polystyrene (SIS65). In linear-viscoelastic measurements $\left(G^{\prime}\right.$ and $\left.G^{\prime \prime}\right)$, below about $180^{\circ} \mathrm{C}$, this copolymer exhibits a low-frequency plateau, a characteristic of microphase separation. Above $180^{\circ} \mathrm{C}, G^{\prime} \sim \omega^{2}$ and $G^{\prime \prime}$ $\sim \omega$ as expected for viscous flow. ${ }^{3,4}$ The effect of shear flow on the structure and properties of SIS65 both above and below the microphase-separation transition (MST) has been studied. The experimental method parallels that described in part 1. A study of the effect of stress on SBS81 was hampered by the stiffness of the sample; only a very narrow range of stress could be attained. In the current work on SIS65 a wider range of stress is possible due to the proximity of the MST; in addition, the polyisoprene (PI) midblock of SIS65 is less entangled than the polybutadiene (PB) block of SBS81: $M_{\mathrm{n}}$ (midblock) $=5 M_{\mathrm{c}}$ instead of $17 M_{\mathrm{c}}$ for SBS81 (see Tables I and II), where $M_{\mathrm{c}}$ is the critical molecular weight for entanglement. ${ }^{5-7}$ The molecular weights of the endblocks in both samples are below the $M_{c}$ of polystyrene.

In part 1 , changes in the linear-viscoelastic properties of SBS81 were correlated with $\chi N$, where $\chi$ is the FloryHuggins interaction parameter and $N$ is the degree of polymerization. This quantity determines the position of the copolymer on the phase diagram. SIS65 differs sufficiently in $\chi N$ from SBS81 to allow us to cross the MST and thus to investigate the effects of flow on a sample sheared near the MST and above $T_{\mathrm{MST}}$.

\section{Experimental Study}

SIS65 was prepared by anionic polymerization with the initiator sec-butyllithium and was stabilized with 2,6-di-tert-butyl-4methylphenol as an antioxidant. The isoprene centerblock is $7.9 \%$ 3,4-polyisoprene by ${ }^{13} \mathrm{C}$ NMR, with the balance being 1,4polyisoprene. The number-average molecular weight was determined by membrane osmometry, $M_{\mathrm{n}}=65000$, and the polydispersity, $M_{\mathrm{w}} / M_{\mathrm{n}}=1.1$, was obtained from gel permeation chromatography. Samples were cast at room temperature from a $10 \%$ toluene solution over approximately 1 week, producing a $1-\mathrm{mm}$-thick film. The films were annealed at $150^{\circ} \mathrm{C}$ for $24 \mathrm{~h}$.

Large-shear strains at constant stress were imposed on the samples in a Rheometrics stress rheometer (RSR) using 25-mmdiameter cone-and-plate fixtures and a nitrogen atmosphere. Samples were subjected to various amounts of stress and strain at several temperatures. When the desired strain was reached, the samples were quenched rapidly to room temperature. At a later time, the samples were inserted into the preheated Rheometrics dynamic spectrometer (RDS) for linear-viscoelastic testing at temperatures from the original test temperature down to 110 ${ }^{\circ} \mathrm{C}$. The ability of this procedure to give reproducible linearviscoelastic results was established in part $1 .^{2}$ Time-temperature superposition allowed for the combination of $G^{\prime}$ and $G^{\prime \prime}$ data at several temperatures into master curves. ${ }^{8}$

After testing in the RDS, the samples were quenched to room temperature where small-angle X-ray scattering (SAXS) was performed. The SAXS measurements were performed at the National Institute of Standards and Technology at Gaithersburg, MD. The camera uses pinhole geometry with a $2 \mathrm{D}$ electronic detector at a sample-to-detector distance of $5.2 \mathrm{~m}$. Small-angle X-ray scattering from the quenched samples reflects the morphology to which the samples have recovered after flow.

Initial Morphology. As-cast samples of SIS65 exhibit a powder-pattern type scattering (Figure 1), implying that the microphase-separated polystyrene cylinders align into grains that are globally randomly oriented. A radial average over all azimuthal angles $\Omega$ yielded the plot of intensity versus $q$ shown in Figure $2(q=(4 \pi / \lambda) \sin \theta ; 2 \theta=$ scattering angle). The positions of the maxima indicate hexagonal packing of the PS cylinders as expected from the composition; arrows indicate the positions of the maxima for a hexagonal lattice with a lattice parameter, 
Table I

Material Parameters for SIS65 and SBS81

\begin{tabular}{lccccccc}
\hline & & \multicolumn{3}{c}{ PS } & & & \\
\cline { 3 - 7 } polymer & $\chi N$ & wt $\%$ & vol $\%$ & $M_{\mathrm{n}}$ & $M_{\mathrm{n}}(\mathrm{PS})$ & $M_{\mathrm{n}}($ rubber $)$ & $M_{\mathrm{n}} / M_{\mathrm{c}}$ \\
\hline SIS65 & $32\left(140^{\circ} \mathrm{C}\right)$ & 25 & 0.236 & 65000 & 8100 & 49000 \\
SBS81 & $24\left(190^{\circ} \mathrm{C}\right)$ & $39\left(160^{\circ} \mathrm{C}\right)$ & 26 & 0.245 & 81300 & 10600 & 60100 \\
& $35\left(180^{\circ} \mathrm{C}\right)$ & & & & &
\end{tabular}

Table II

Material Parameters for the Constituent Homopolymers

\begin{tabular}{ccccc}
\hline polymer & $M_{\mathrm{c}}$ & $T_{\mathrm{g}},{ }^{\circ} \mathrm{C}$ & $\rho, \mathrm{g} / \mathrm{cm}^{3}$ & \multicolumn{1}{c}{$\chi(\mathrm{PS})$} \\
\hline PS & 37400 & 100 & 1.04 & \\
PI & 10200 & -68 & 0.899 & $0.08\left(140^{\circ} \mathrm{C}\right)^{6}$ \\
& & & & $0.06\left(190^{\circ} \mathrm{C}\right)$ \\
PB & 3600 & -67 & 0.895 & $0.07\left(160^{\circ} \mathrm{C}\right)^{7}$ \\
& & & & $0.06\left(180^{\circ} \mathrm{C}\right)$
\end{tabular}

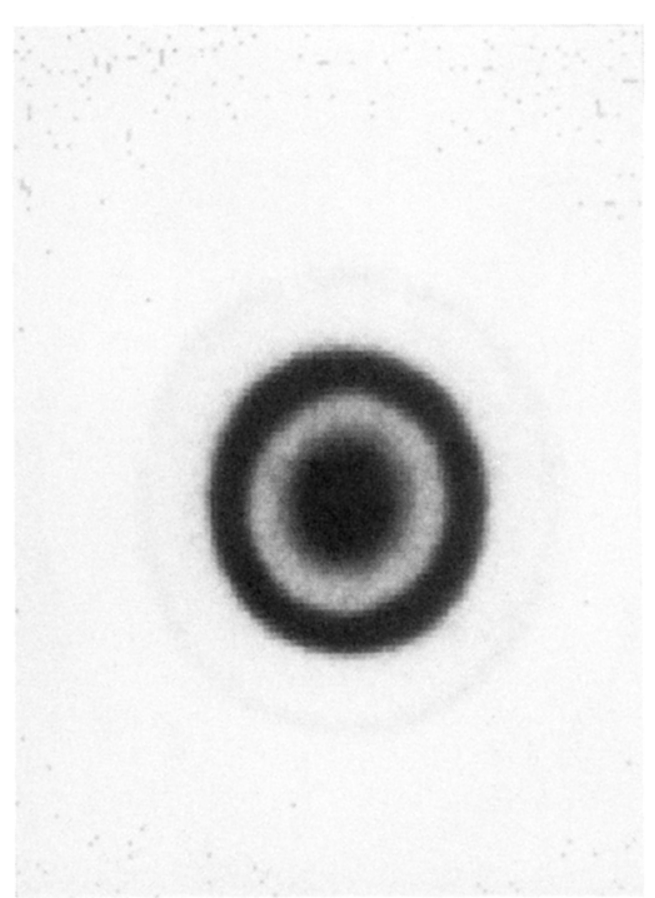

Figure 1. SAXS pattern of the cast polymer.

$a$, of $246 \AA$. The locations of the experimental maxima are shown in Table III along with the same information obtained previously for SBS81. The size of the hexagonal unit cell together with volume considerations determines the diameter of the PS domains

$$
d=a\left\{\frac{2 \phi_{\mathrm{PS}} \sqrt{3}}{\pi}\right\}^{1 / 2}=126 \AA
$$

where $\phi_{\mathrm{PS}}$ is the volume fraction of PS.

The second contribution to the scattering is the form factor, in this case the form factor for infinitely long cylinders. This function has also been superimposed on Figure 2 for a cylinder diameter of $126 \AA$. The strong minimum in the form factor falls at the same position as the third predicted maxima of the hexagonal array. This minimum, although a potentially dominant feature, is not observed due to peak broadening from disorder and also due to the overall weakness of scattering at this scattering vector.

Linear-viscoelastic moduli curves, $G^{\prime}$ and $G^{\prime \prime}$, were taken between 110 and $195^{\circ} \mathrm{C}$ and $0.1 \leq \omega \leq 20 \mathrm{rad} / \mathrm{s}$ at a strain amplitude of 0.03 . The results were combined into master curves (Figure 3 ) by shifting the frequency axis by an amount $a_{\mathrm{T}}$; the shift factors are plotted versus inverse temperature in Figure 4. The moduli of SIS65 form two distinct master curves: one for $T<180^{\circ} \mathrm{C}$ and the other for $T \geq 180^{\circ} \mathrm{C}$. The low-temperature master curve is typical of a microphase-separated block copolymer. At high frequencies $G^{\prime}$ approaches a plateau equivalent to the entanglement plateau of polyisoprene, $4.4 \times 10^{5} \mathrm{~Pa}$. At low

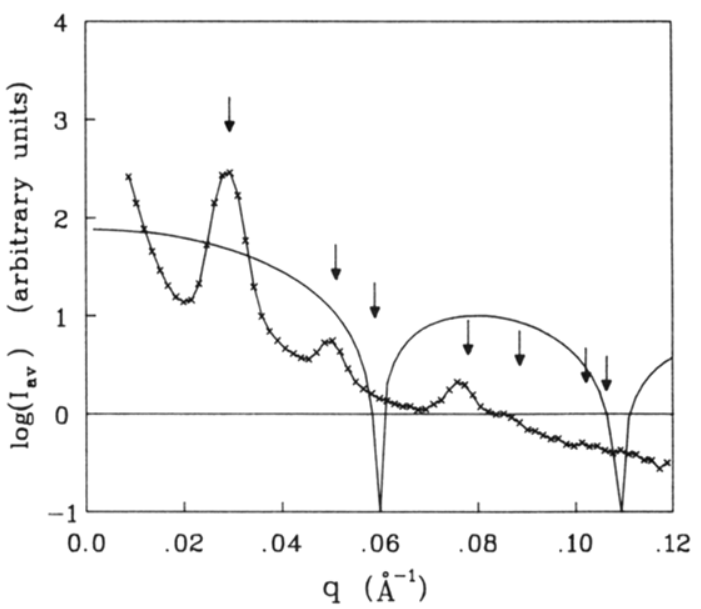

Figure 2. Circular average over all azimuthal angles $\Omega$ of the SAXS pattern of the cast film $(x)$. The locations of the maxima in a hexagonal lattice scattering pattern for $a=246 \AA$ are indicated with arrows. Also shown (-) is the form factor scattering for infinite cylinders of diameter $126 \AA$.

Table III

Lattice Spacings and Parameters for both SIS65 and SBS81

\begin{tabular}{cccc}
\hline & \multicolumn{3}{c}{$q_{10} / q_{h k}$} \\
\cline { 2 - 4 } $\begin{array}{c}\text { lattice plane } \\
(h k)\end{array}$ & $\begin{array}{c}\text { hexagonal } \\
\text { lattice }\end{array}$ & SBS81 & SIS65 \\
\hline 10 & 1 & 1 & 1 \\
11 & 0.577 & 0.587 & 0.588 \\
20 & 0.500 & & \\
21 & 0.378 & 0.378 & 0.382 \\
$a, \AA$ & & 284 & 246 \\
$d, \AA$ & & 148 & 126
\end{tabular}

frequencies there is the beginning of a power law region $\left(G^{\prime} \sim\right.$ $\left.G^{\prime \prime} \sim \omega^{n}, n \sim 0.4\right)$, indicating the existence of microphase separation in the system. $3,4,9$ Above $180^{\circ} \mathrm{C}$ a different master curve is formed in which $G^{\prime}$ and $G^{\prime \prime}$ are several orders of magnitude smaller than at low temperatures and exhibit the classical behavior of a polymer liquid with $G^{\prime} \sim \omega^{2}$ and $G^{\prime \prime} \sim$ $\omega$.

Flow in the Microphase-Separated State. The as-cast samples were sheared at constant shear stress below $T_{\text {MST }}$ up to prescribed strains. A temperature of $140^{\circ} \mathrm{C}$ was chosen to ensure microphase separation in the system. It should be noted that SIS65 is considerably softer than SBS81, an indication of its proximity to the MST and its less entangled state. A shear stress of $2000 \mathrm{~Pa}$ required a rate of about $2 \mathrm{~s}^{-1}$ in SIS65 as compared to $10^{-3} \mathrm{~s}^{-1}$ in SBS81. This implied that the shearing times were very different for the two samples: about $1 \mathrm{~min}$ for SIS65 versus $60 \mathrm{~min}$ for SBS81. Two studies were made: one on the effect of varying stress at constant strain and the other on the effect of varying strain at constant stress.

Effect of Stress. The two-dimensional SAXS patterns of SIS65 samples subjected to a strain of $\gamma=20$ at two different stresses, $\tau=20$ and $2000 \mathrm{~Pa}$, at $140^{\circ} \mathrm{C}$ are shown in Figure 5 . The flow direction is indicated. The angle $\Omega$ describes the azimuthal dependence of the scattering; $\Omega=0$, the line of principal scattering, will be referred to as the equator and $\Omega=\pi / 2$ as the meridian (coincident with the flow direction). The SAXS pattern for $\tau=20 \mathrm{~Pa}$ (Figure 5a) is nearly isotropic while for $\tau=2000$ 


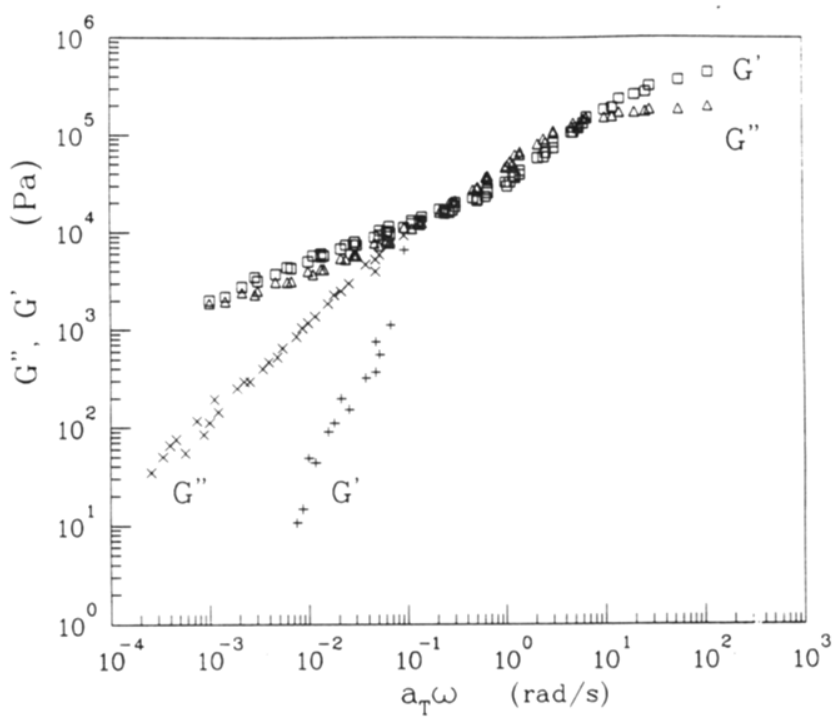

Figure 3. Linear-viscoelastic master curves of SIS65. $T \geq 180$ ${ }^{\circ} \mathrm{C}: G^{\prime}(+), G^{\prime \prime}(\times) . T<180^{\circ} \mathrm{C}: G^{\prime}(\square), G^{\prime \prime}(\Delta) . T_{\text {ref }}=110^{\circ} \mathrm{C}$.

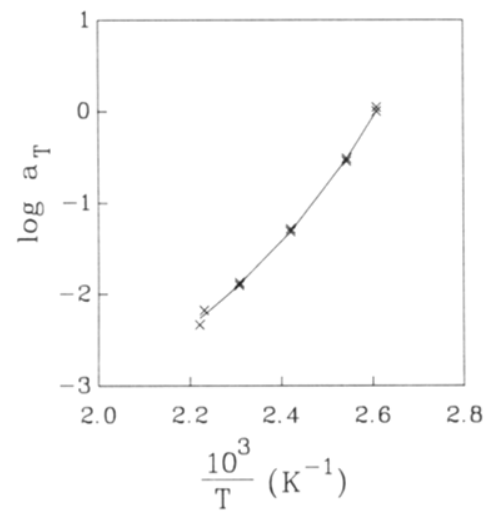

Figure 4. Empirical shift factors, $a_{\mathrm{T}}$, versus inverse temperature: $T_{\text {ref }}=110^{\circ} \mathrm{C}$. Also shown $(-)$ is the fit to the WLF equation for $C_{1}=0.19$ and $C_{2}=93.2 \mathrm{~K}$.

$\mathrm{Pa}$ (Figure 5b) most of the intensity is concentrated on the equator, indicating substantial orientation. Two spots at low $q$ in the equatorial scattering of the $\tau=20 \mathrm{~Pa}$ sample are unique to this otherwise isotropic pattern and are due to anomalous parasitic scattering.

Ten degree sector averages around the equator and the meridian are compared in Figures 6 and 7. Recall that both samples were sheared to the same strain magnitude, $\gamma=20$. The positions of the maxima are unchanged from those of the unoriented material, which implies that the diameter of the PS microdomains is not changed by the shear treatment. The higher amount of orientation at higher stress is particularly visible at $\Omega=\pi / 2$ where there is very little intensity for the high-stress sample compared with that of the low-stress sample.

Effect of Strain. To determine the effect of total strain magnitude $\gamma$, samples were subjected to various strains at a common stress, $\tau=2000 \mathrm{~Pa}$ at $140^{\circ} \mathrm{C}$. This stress is sufficient to produce orientation in both SIS65 and SBS81. ${ }^{2}$ The resulting SAXS patterns are shown in Figure 8, with the positions of the maxima again unchanged from those of the unoriented material. The distribution of scattering over the angle $\Omega$ becomes anisotropic with increasing strain. As a measure of microdomain orientation, the ratio of equatorial to meridional scattering, $I_{\mathrm{e}} /$ $I_{\mathrm{m}}$, at $q=q_{\max }$ has been plotted (Figure 9). The orientation increases quickly at first and ultimately reaches a saturation level at high strains at about $\log \left(I_{\mathrm{e}} / I_{\mathrm{m}}\right) \sim 2$. These results are compared with those for SBS81 in Figure 9.

The master curves of dynamic moduli for SIS65 are shown in Figure 10 for all strains. $G^{\prime}$ and $G^{\prime \prime}$ decrease with increasing strain at all frequencies until saturation is reached. For small strains, this observation agrees with the earlier experiments on SBS81.
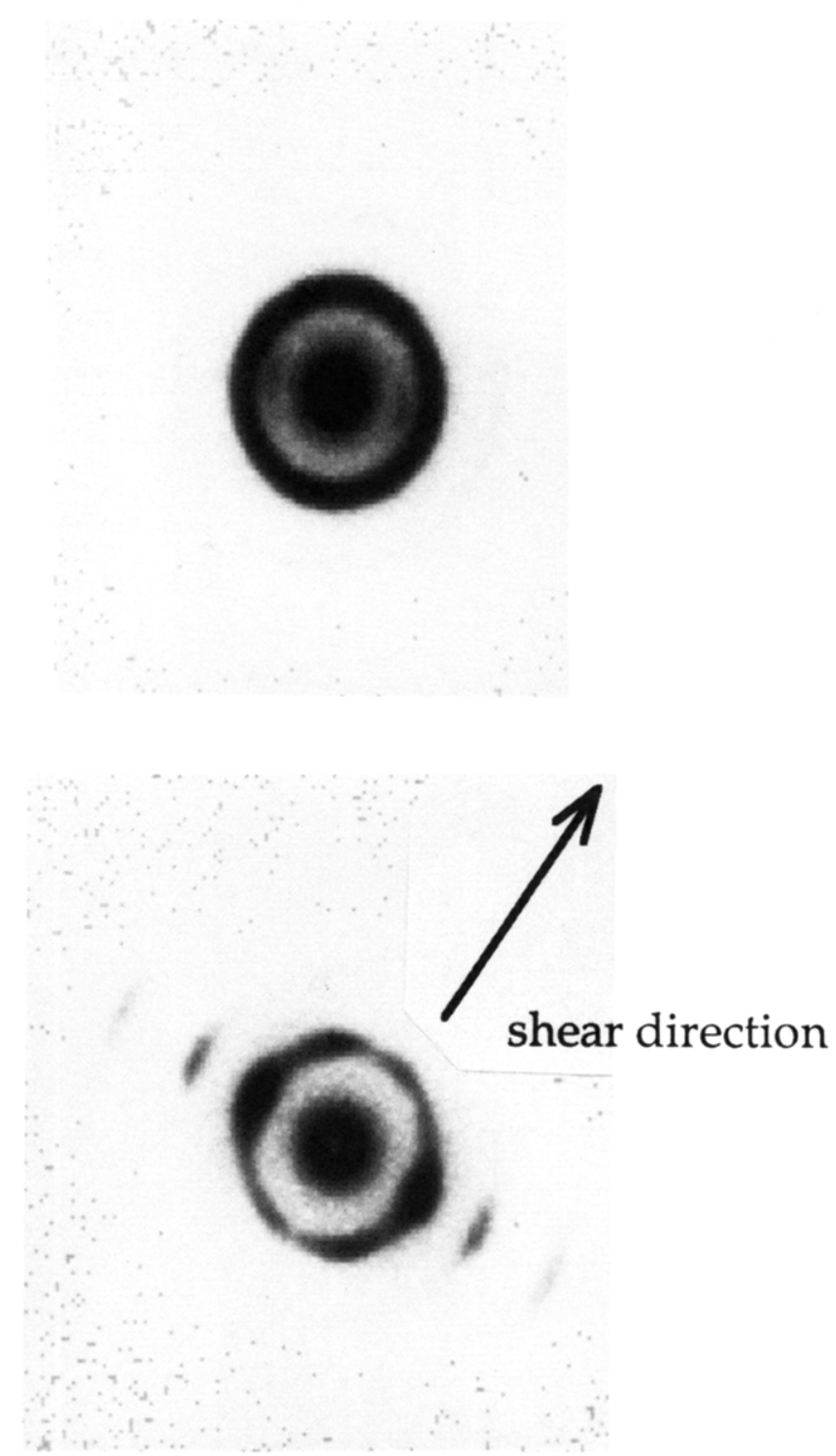

Figure 5. SAXS patterns of samples strained to $\gamma=20$ at $T=$ $140{ }^{\circ} \mathrm{C}$ at $\tau=$ (a) 20 and (b) $2000 \mathrm{~Pa}$.

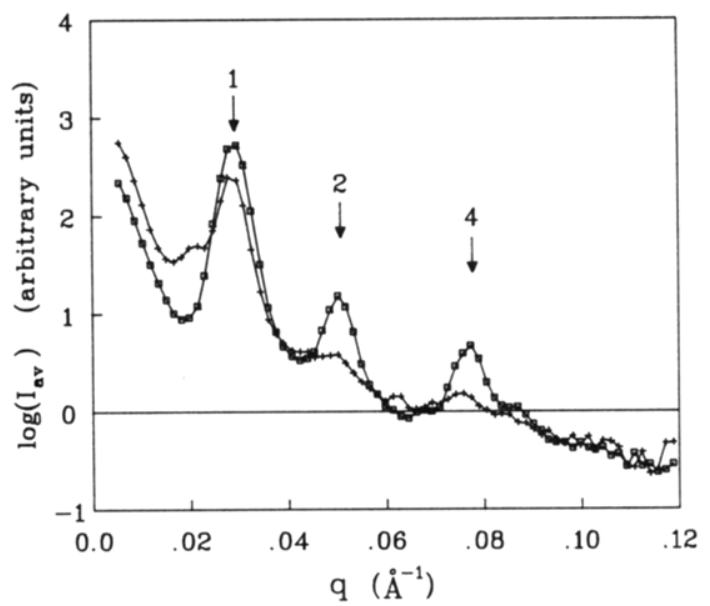

Figure 6. SAXS patterns of samples strained to $\gamma=20$ at $T=$ $140^{\circ} \mathrm{C}$ at $\tau=(+) 20$ and (口) $2000 \mathrm{~Pa}$. Sector average over $10^{\circ}$ along equator $(\Omega=0)$.

However, for SBS81 the trend reversed at higher strains, and $G^{\prime}$ and $G^{\prime \prime}$ increased with increasing strain. For SIS65 at high strains only a slight increase in moduli is observed at the highest frequencies. The initial entanglement plateau is not recovered, although the strain imposed is much higher than that achieved 


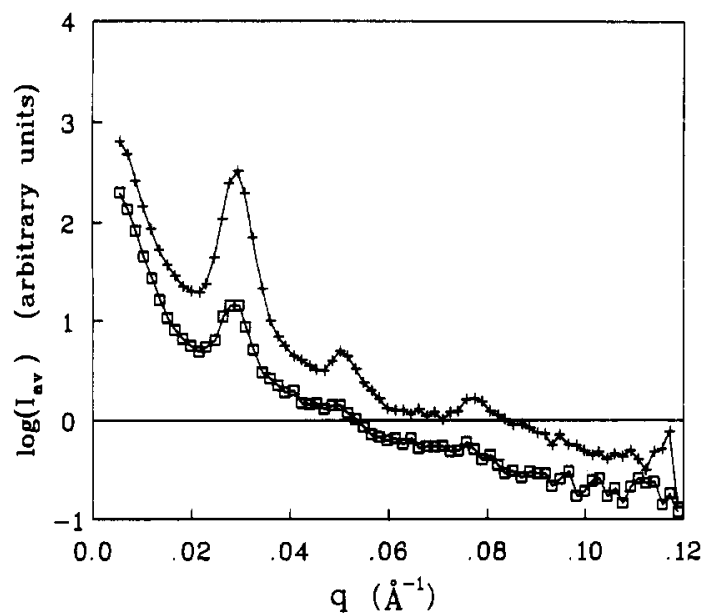

Figure 7. SAXS patterns of samples strained to $\gamma=20$ at $T=$ $140^{\circ} \mathrm{C}$ at $\tau=(+) 20$ and (a) $2000 \mathrm{~Pa}$. Sector average over $10^{\circ}$ along meridian $(\Omega=\pi / 2)$.

for SBS81. Thus, although the orientation measurements are comparable for the two materials, the effect of shear on the dynamic mechanical properties is different.

Shearing in the Homogeneous State. The accessibility of $T_{\text {MST }} \sim 180^{\circ} \mathrm{C}$ for SIS65 makes it possible to investigate the effect of flow in the homogeneous regime; a structuring experiment was performed at $190^{\circ} \mathrm{C} ; \tau=2000 \mathrm{~Pa}$ to a total strain of $\gamma=63$. As can be seen in the SAXS pattern in Figure 11, the resulting sample is nearly isotropic. The degree of orientation can be evaluated from the equatorial and meridional scattering averages in Figure $12\left(10^{\circ}\right.$ sector averages). There is very little difference between these two curves and the circular average of the unoriented sample of SIS65. The lattice spacings are the same as those for all other patterns. Although the sample was subjected to a flow that in terms of stress and strain should have produced structure, very little anisotropy is seen. It appears that the existence of microphases in the melt is necessary for PS microdomains to become oriented.

\section{Discussion}

Under the appropriate conditions, the influence of flow on SIS65 is the same as that for SBS81: increases in either stress or strain increase the microdomain orientation. The mechanism proposed previously is applicable: at high enough stress and strain, the flow disrupts the microdomain structure, and the partially disrupted and reformed microdomains align in the flow field. The flow energy, $\tau \gamma$, acts to counterbalance the free energy of mixing, $\Delta G_{\text {mix }}$, which can be estimated from Flory-Huggins theory ${ }^{10}$

$$
\Delta G_{\text {mix }}=R T\left(\frac{\phi_{\mathrm{PS}} \ln \phi_{\mathrm{PS}}}{V_{\mathrm{PS}} N_{\mathrm{PS}}}+\frac{\phi_{\mathrm{PI}} \ln \phi_{\mathrm{PI}}}{V_{\mathrm{PI}} N_{\mathrm{PI}}}+\frac{\chi \phi_{\mathrm{PS}} \phi_{\mathrm{PI}}}{V_{\text {ref }}}\right)
$$

where $\phi_{\mathrm{PI}}$ is the volume fraction of polyisoprene, $R$ is the gas constant, $V_{\mathrm{PS}}$ and $V_{\mathrm{PI}}$ are the molar volumes of $\mathrm{PS}$ and PI, $N_{\mathrm{PS}}$ and $N_{\mathrm{PI}}$ are the molar volumes of PS and PI, $N_{\mathrm{PS}}$ and $N_{P I}$ are the degrees of polymerization of the two blocks, and $V_{\text {ref }}$ is the reference volume for $\chi$, which is taken to be ${ }^{11,12}$

$$
V_{\text {ref }}=\left(V_{\mathrm{PS}} V_{\mathrm{PI}}\right)^{1 / 2}
$$

It is not implied here that we believe that the copolymer microphases mix completely during flow. On the contrary, we believe that the disruption that occurs is the minimum necessary to allow the flow. We use $\Delta G_{\text {mix }}$ only as the upper bound on the amount of energy put into the system to cause flow. For SIS65 at $140^{\circ} \mathrm{C}, \Delta G_{\operatorname{mix}} \sim 5 \times 10^{5} \mathrm{~Pa}$. At high stress, $\tau \gamma=4 \times 10^{4} \mathrm{~Pa}$, and the flow energy approaches that necessary to homogenize the copolymer. Thus, some disruption of microdomains could be expected, and the situation is analogous to that seen in part 1.
The low-stress situation is different, however, since $\tau \gamma$ $=400 \mathrm{~Pa}$, and microdomain disruption does not seem likely at this energy level. The previously described flow mechanism would predict that no flow would occur until the domains begin to disrupt. Flow does occur, however, and to high strains, only a small fraction of which is recoverable. Morphological measurements indicate that the low-stress flow did not produce orientation, however. One possible explanation is that, at stresses that are too low to disrupt the structure, grains of well-aligned microdomains flow as units, leaving the microdomains themselves intact. Such a mechanism was rejected for the flow of SBS81 because tumbling grains would not result in global microdomain orientation, but, in the case of SIS65 at $20 \mathrm{~Pa}$, orientation is not observed. One flow that can be proposed, then, involves slippage along grain boundaries, without microdomain disruption, producing a random orientation of PS microdomains. This kind of flow can also be described as the pulling out of a small fraction of the PS segments-just enough to allow flow. Thus, the system is never very disrupted, but it flows.

Above $T_{\mathrm{MST}}, \Delta G_{\operatorname{mix}}\left(190^{\circ} \mathrm{C}\right)$ is estimated to be $4 \times 10^{5}$ $\mathrm{Pa}$, and for the flow imposed, $\tau \gamma \sim 1.3 \times 10^{5} \mathrm{~Pa}$. It is known from linear-viscoelastic measurements that at 190 ${ }^{\circ} \mathrm{C}$ SIS65 is in the homogeneous state. In this region, orientation is not produced in this sample, even at high stress and strain. This confirms that the microdomain dissolution which occurs below the MST does not lead to complete phase compatibility since in that case microdomain orientation would not be observed. It is therefore important to realize that some degree of microphase separation is required in order for the flow to have an influence on the microdomain orientation. A disrupted, but microphase-separated, structure is compatible with these observations.

Interpretation of the Rheological Data. For SBS81 the increase in $G^{\prime}$ and $G^{\prime \prime}$ at high frequencies was interpreted as a result of the ability of the flowing material to reform a well-microphase-separated network upon cessation of shear once the old network is sufficiently disrupted and before long-range motion is suppressed by microphase separation. The original phase structure is disrupted by the flow, and when the flow stops, the microphase separation is reestablished. The extent to which the original degree of microphase separation is reestablished depends on the trade-off between the mobility of the chains and the desire to microphase separate. The desire to microphase separate was very high for SBS81, and when the mobility was also very high $\left(T=180^{\circ} \mathrm{C}\right)$, the original morphology was reestablished.

The difference in the rheological behavior of SIS65 when compared with that of SBS81 is due to differences in two areas: the magnitude of the microphase-separation energy and the number of entanglements. For both SIS65 and SBS81 the cast network is thought to be dissolved by highstress flow, and upon cessation of shear the material is under two energy constraints: to relax elastic energy and to satisfy microphase separation. For SIS65 the desire to microphase separate is low. Without a strong desire to reform the well-microphase-separated domains, the material will not achieve a high degree of order. Thus $G^{\prime}$ and $G^{\prime \prime}$ decrease with increasing shear strain, but instead of recovering to higher values with high strain as was seen for the strongly microphase-separating SBS81, SIS65 does not recover and saturates at low values of $G^{\prime}$ and $G^{\prime \prime}$. The degree of microphase separation of these materials is thus permanently altered by flow. 

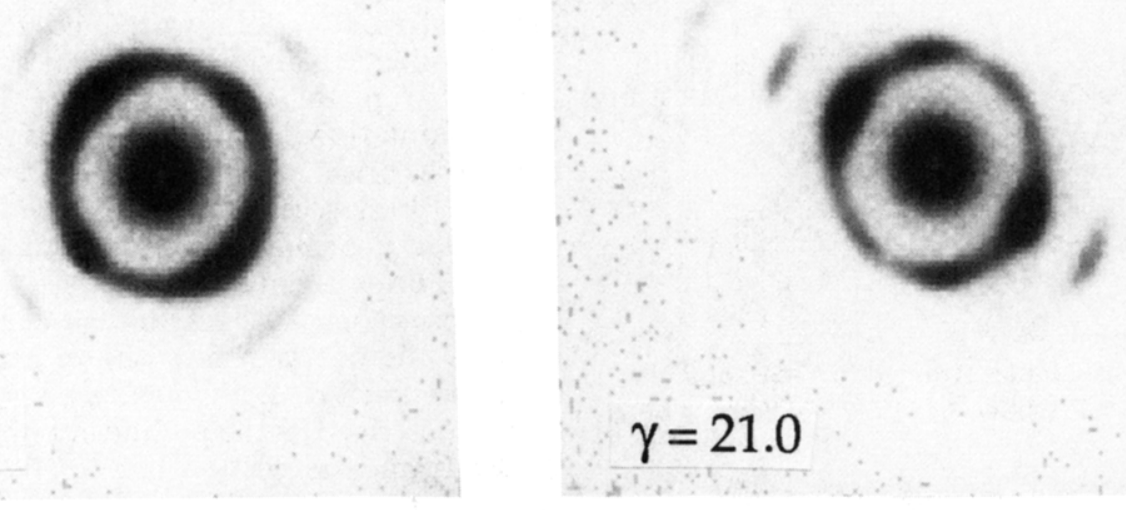

$\gamma=5.5$
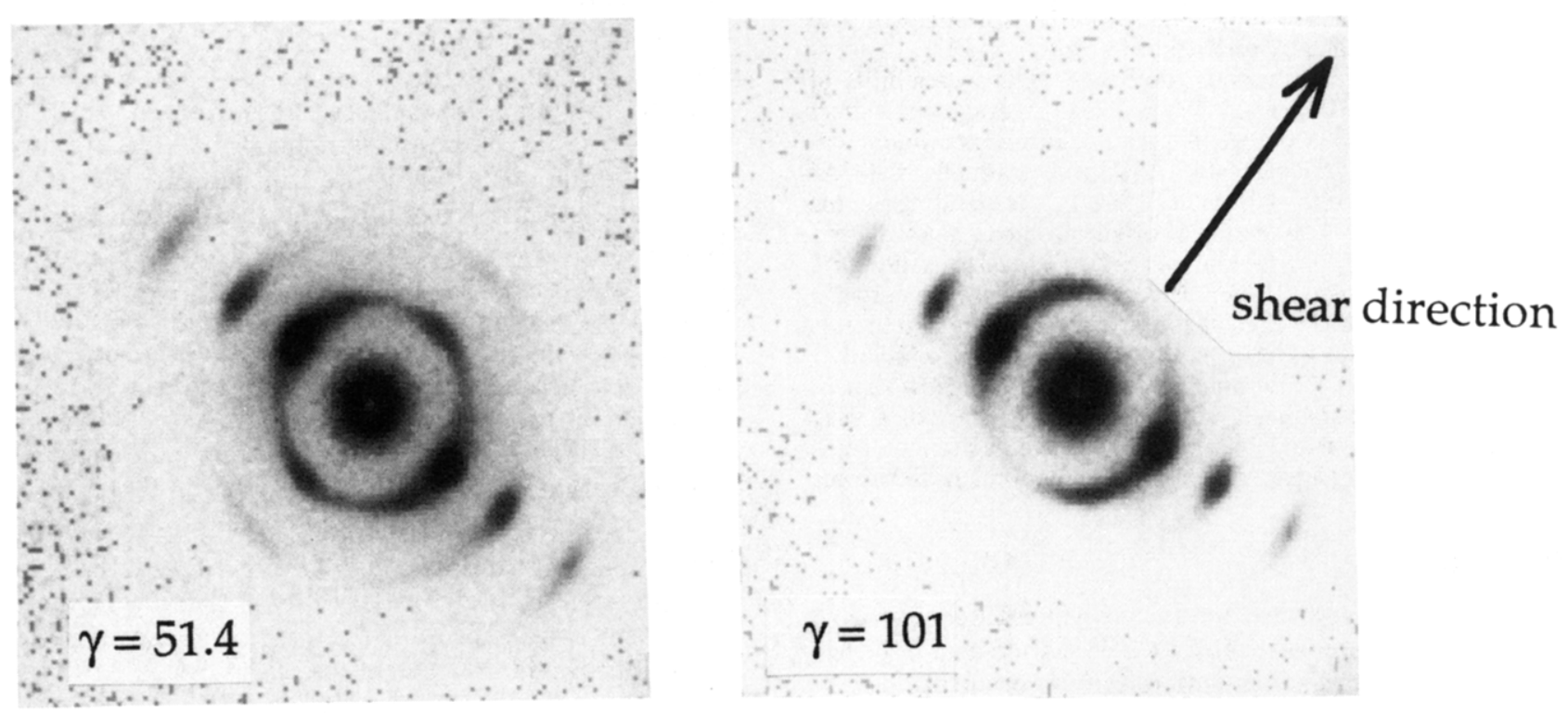

$$
\gamma=101
$$
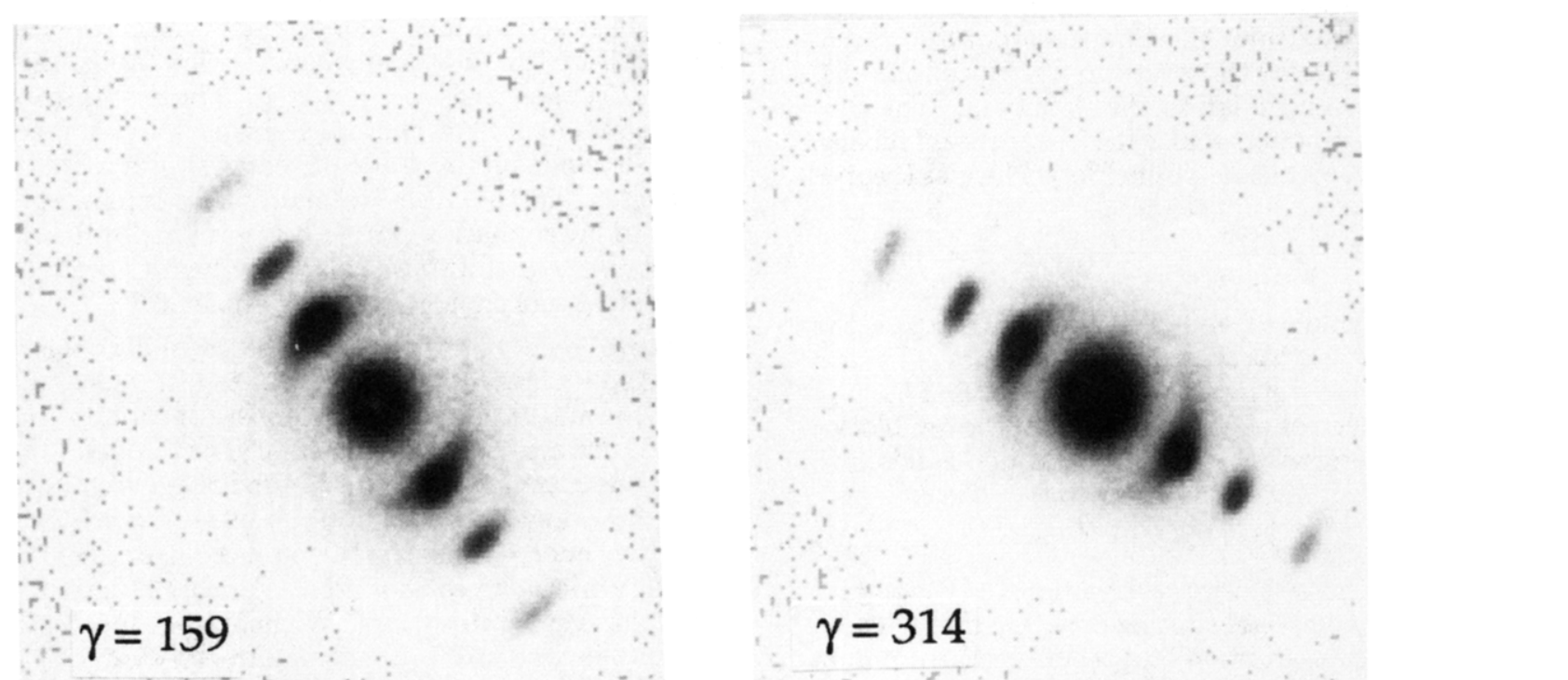

Figure 8. SAXS of samples strained at $\tau=2000 \mathrm{~Pa}, T=140^{\circ} \mathrm{C}$, to strains of (a) 5.5 , (b) 21.0 , (c) 51.4 , (d) 101 , (e) 159 , and (f) 314 .

The molecular weight between entanglements is also quite large for PI as compared with that for PB. This reflects a more relaxed, open network structure-and thus lower elastic forces when perturbed. As in the case of mi- crophase-separation energy, the chains are not strongly driven to reentangle, and the entanglement plateau remains depressed after the flow.

Our interpretation of the measurements on SIS65 can 


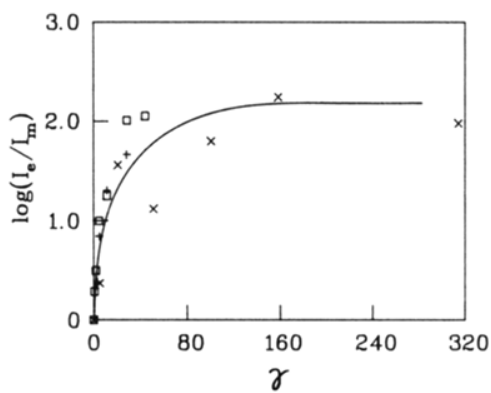

Figure 9. $\log$ of the ratio of equatorial to meridional scattering of SBS81 at $T=(\square) 160^{\circ} \mathrm{C}$ and $(+) 180^{\circ} \mathrm{C}$ at $q=0.0261 \AA^{-1}$ and of SIS65 at $T=140^{\circ} \mathrm{C}(\times)$ at $q=0.0295 \AA^{-1}$.

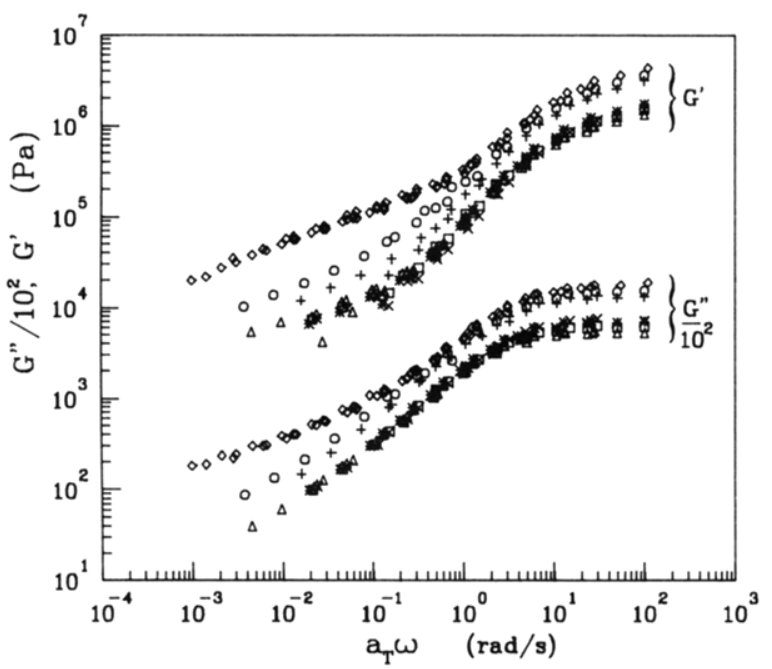

Figure 10. Master curves of $G^{\prime}$ and $G^{\prime \prime} / 100$ for samples sheared at $\tau=2000 \mathrm{~Pa}, T=140^{\circ} \mathrm{C}$, to $\gamma=(\diamond) 0,(0) 5.5,(+) 21$, (口) $51.4,(\Delta) 101,\left(^{*}\right) 159$, and $(\times) 314 ; T_{\text {ref }}=110^{\circ} \mathrm{C}$.

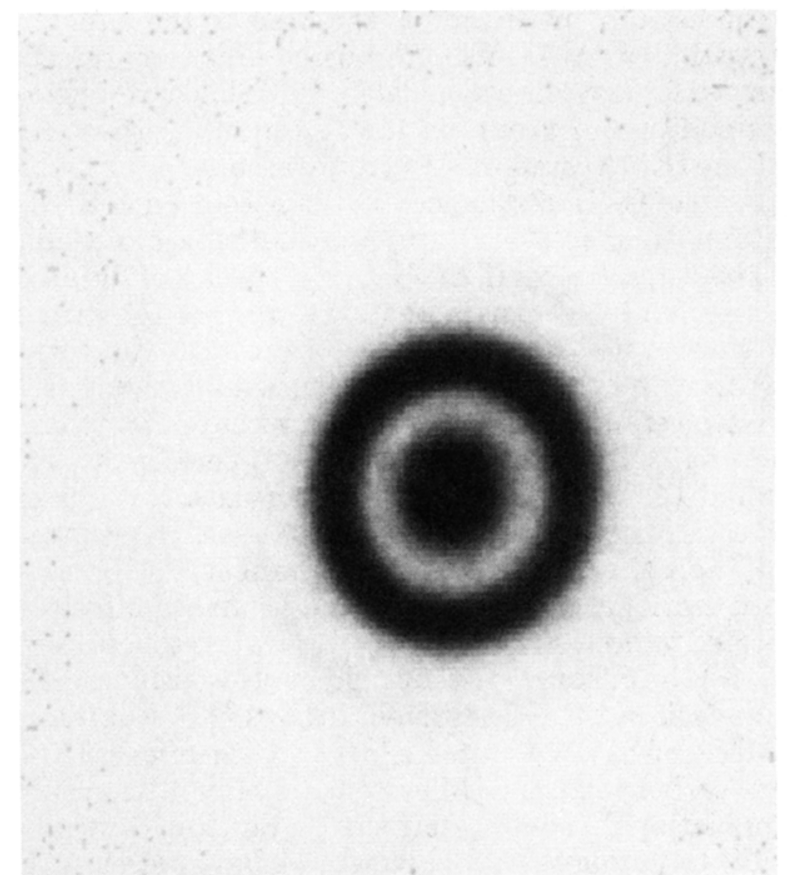

Figure 11. SAXS of sample sheared to $\gamma=63$ at $\tau=2000 \mathrm{~Pa}$ and $T=190^{\circ} \mathrm{C}$.

be summarized in the following picture of flow-induced structuring of microphase-separated block copolymers:

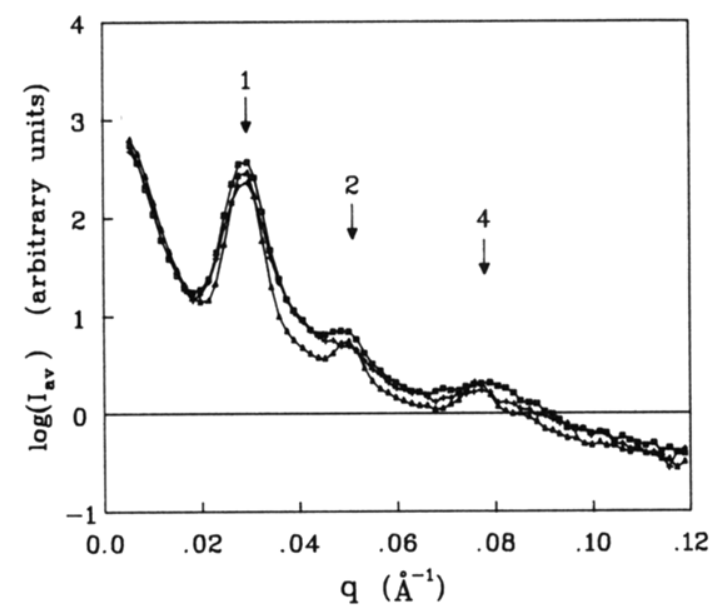

Figure 12. SAXS of sample sheared to $\gamma=63$ at $\tau=2000 \mathrm{~Pa}$ and $T=190^{\circ} \mathrm{C}$. Sector averages for $(+) \Omega=0$ and $(\square) \Omega=\pi / 2$. Also included is the circular average of the unoriented sample $(\Delta)$.

First, shearing above a critical stress introduces a global domain orientation in the shear direction. Below that stress the domains are not disrupted, and the flow of grains occurs; this type of flow does not result in domain orientation. Second, the formation of a highly oriented microdomain structure is dependent on a microphaseseparated flow morphology as demonstrated by the structuring experiment above $T_{\text {MST }}$. Third, the degree of phase mixing in the final material will depend on the strain that the system has experienced as well as the microphaseseparation energy. Further experiments are needed to determine the changes in the degree of microphase separation for both SBS81 and SIS65 as a test of the proposed structuring mechanism.

Acknowledgment. We are grateful to the Air Force Office of Scientific Research and the Office of Naval Research for the funding of this study. The Rheometrics stress rheometer was made available to us by Rheometrics, Inc. We thank Ned Thomas for his advice on the interpretation of the SAXS results and Xina Quan, Ron Larson, and Frank Bates for their comments on the manuscript.

\section{References and Notes}

(1) Current address: Department of Chemistry and Chemical Engineering, Michigan Technological University, Houghton, MI 49931.

(2) Morrison, F. A.; Winter, H. H. Macromolecules 1989, 22, 3533.

(3) Chung, C. I.; Gale, J. C. J. Polym. Sci., Polym. Phys. Ed. 1976, 14,1149 .

(4) Gouinlock, R. V.; Porter, R. S. Polym. Eng. Sci. 1977, 17, 535.

(5) Friedrich, S. M.S. Thesis, University of Massachusetts, Amherst, MA, 1984.

(6) Rounds, N. A. Ph.D. Thesis, University of Akron, Akron, OH, 1970.

(7) Ronca, G.; Russell, T. P. Phys. Rev. B 1987, 35, 8566.

(8) Ferry, J. D. Viscoelastic Properties of Polymers; 3rd ed.; John Wiley \& Sons: New York, 1980.

(9) Bates, F. S. Macromolecules 1984, 17, 2607.

(10) Flory, P. J. Principles of Polymer Chemistry; Cornell University Press: Ithaca, NY, 1953.

(11) Helfand, E. Macromolecules 1975, 8, 552.

(12) Roe, R.-J.; Zin, W.-C. Macromolecules 1980, 13, 1221.

Registry No. (PS)(PI) (block copolymer), 105729-79-1. 